\title{
Investigation of nonlinear absorption processes with femtosecond light pulses in lithium niobate crystals
}

\author{
O. Beyer,* D. Maxein, and K. Buse \\ Institute of Physics, University of Bonn, Wegelerstr. 8, D-53115 Bonn, Germany \\ B. Sturman \\ Institute of Automation and Electrometry, 630090 Novosibirsk, Russia \\ H. T. Hsieh and D. Psaltis \\ Department of Electrical Engineering, California Institute of Technology, Pasadena, California 91125, USA
}

(Received 20 December 2004; published 10 May 2005)

\begin{abstract}
The propagation of high-power femtosecond light pulses in lithium niobate crystals $\left(\mathrm{LiNbO}_{3}\right)$ is investigated experimentally and theoretically in collinear pump-probe transmission experiments. It is found within a wide intensity range that a strong decrease of the pump transmission coefficient at wavelength $388 \mathrm{~nm}$ fully complies with the model of two-photon absorption; the corresponding nonlinear absorption coefficient is $\beta_{p}$ $\simeq 3.5 \mathrm{~cm} / \mathrm{GW}$. Furthermore, strong pump pulses induce a considerable absorption for the probe at $776 \mathrm{~nm}$. The dependence of the probe transmission coefficient on the time delay $\Delta t$ between probe and pump pulses is characterized by a narrow dip (at $\Delta t \simeq 0$ ) and a long (on the picosecond time scale) lasting plateau. The dip is due to direct two-photon transitions involving pump and probe photons; the corresponding nonlinear absorption coefficient is $\beta_{r} \simeq 0.9 \mathrm{~cm} / \mathrm{GW}$. The plateau absorption is caused by the presence of pump-excited charge carriers; the effective absorption cross section at $776 \mathrm{~nm}$ is $\sigma_{r} \simeq 8 \times 10^{-18} \mathrm{~cm}^{2}$. The above nonlinear absorption parameters are not strongly polarization sensitive. No specific manifestations of the relaxation of hot carriers are found for a pulse duration of $\simeq 0.24$ ps.
\end{abstract}

DOI: 10.1103/PhysRevE.71.056603

PACS number(s): 42.50.Gy, 77.84.Dy, 78.20.Bh, 78.47.+p

\section{INTRODUCTION}

Femtosecond high-power light pulses have become increasingly important in the physics of light-matter interactions. A crucial advantage of such pulses is the possibility to deposit rapidly and precisely light energy in solids. A direction of femtosecond studies is the characterization of carrier and phonon dynamics in semiconductors [1-3]. The fundamental items include here momentum and energy relaxation, the carrier-carrier scattering, intervalley transitions, and carrier diffusion. Various experimental methods have been developed for probing ultrafast dynamics [1]. They include pump-and-probe techniques, streak cameras, and optical Kerr gates. Numerous publications (see $[4,5]$ and references therein) deal with the determination of Kerr and two-photon absorption coefficients in wide-gap optical materials.

Ferroelectric crystals, such as $\mathrm{LiNbO}_{3}, \mathrm{BaTiO}_{3}$, and $\mathrm{KNbO}_{3}$, hold an intermediate position between wide-gap materials (glasses) and semiconductors with respect to the value of the forbidden gap. They are transparent in the visible and an interplay between nonlinear effects of a different nature should take place with increasing light intensity. These include the instantaneous $\chi^{(2)}$ and $\chi^{(3)}$ nonlinearities, two-photon absorption, and inertial free-carrier and photorefractive nonlinearities. A physical picture of nonlinear phenomena in the femtosecond pulse range is practically absent.

\footnotetext{
*Electronic address: beyer@physik.uni-bonn.de; www.physik.uni-bonn.de/hertz
}

URL:
The data on electron transitions and charge transport, accumulated in continuous-wave and long-pulse holographic experiments (see, e.g. [6-11]), form a background for femtosecond studies.

In this paper we present the results of our experimental and theoretical studies of the light-induced processes in lithium niobate $\left(\mathrm{LiNbO}_{3}\right)$ crystals on the subpicosecond $\left(\simeq 10^{-13} \mathrm{~s}\right)$ time scale. The choice of this material is not accidental. It is of prime importance for various nonlinear and photorefractive applications including frequency conversion, optical parametric oscillation, holographic data storage, and domain engineering (see, e.g., [12-18]).

Information about the response of $\mathrm{LiNbO}_{3}$ crystals to high-power light pulses, available in the literature, is scarce. The literature values of the two-photon absorption coefficient $\beta$ range over more than one order of magnitude at the same wavelength $(\approx 530 \mathrm{~nm})$ [19]. These results were obtained utilizing nanosecond and subnanosecond light pulses. Most probably, the large scatter of the data is caused by systematic errors resulting from additional absorption effects from excited charge carriers that are unavoidable for long highpower pulses [5]. These processes are known to be very important for lithium niobate [20]. The only available literature data of the Kerr coefficient $n^{(2)}$ refer to the wavelength $\lambda$ $=532 \mathrm{~nm}$ [4]; it was also obtained with the use of long pulses.

\section{EXPERIMENTAL ARRANGEMENT}

In our experiments we use a pump-and-probe technique. An axially symmetric pulse at the wavelength $\lambda_{r}=776 \mathrm{~nm}$ is 


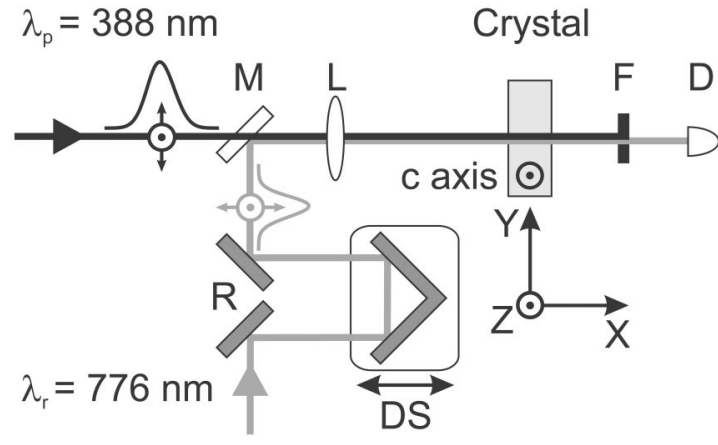

FIG. 1. Schematic diagram of a collinear pump-probe experiment; $\mathrm{M}$ is a dichroic mirror, $\mathrm{L}$ is a long-focus $(500 \mathrm{~mm})$ lens, $\mathrm{F}$ is a band edge filter for the pump light, D is a photodetector, DS is a probe delay stage, and $\mathrm{R}$ are reflectors.

obtained from a Ti:sapphire amplified laser system CPA2010, Clark-MXR, Inc. The temporal full width at half maximum (FWHM) of the pulse intensity is $\simeq 0.24$ ps. About $4 \%$ of the pulse energy is tapped and serves as the incident probe. The rest of the pulse is passed through a 1-mm-thick BBO $\left(\beta-\mathrm{BaB}_{2} \mathrm{O}_{4}\right)$ crystal to generate a pump pulse at the wavelength $\lambda_{p}=388 \mathrm{~nm}$. The remaining $776 \mathrm{~nm}$ light in the pump beam is blocked with the help of dielectric filters.

The experimental setup is shown schematically in Fig. 1. Pump and probe pulses are incident normally and collinearly onto the $Y Z$ face of a $\mathrm{LiNbO}_{3}$ sample. The arrival time of the probe pulse can be tuned by a variable-delay stage. The polar c axis of the sample is parallel to the $Z$ axis. The input polarization vector of the pump pulse is either parallel or perpendicular to the c axis. The spatial FWHM of the pulses at the input face is about $0.6 \mathrm{~mm}$. The peak intensity of the pump pulse $I_{p}^{0}$ ranges from $\simeq 1$ to $\simeq 276 \mathrm{GW} / \mathrm{cm}^{2}$. The maximum pump pulse fluence is about $59 \mathrm{~mJ} / \mathrm{cm}^{2}$. The output energy of the pump and probe pulses can be measured separately by a photodetector.

The difference in the wavelengths $\lambda_{r}$ and $\lambda_{p}$ enables us to separate easily the transmitted pump and probe signals. On the other hand, the collinear propagation of the pump and probe pulses simplifies the modelling procedures.

Four different samples of $\mathrm{LiNbO}_{3}$, labeled as 1, 2, 3, and 4 , are used in our experiments. The values of the thickness $d$ for them are 1, 0.5, 0.07, and $0.07 \mathrm{~mm}$, respectively. Samples 1, 2, and 3 are nominally undoped and possess very small $\left(\alpha_{0} \lesssim 0.1 \mathrm{~cm}^{-1}\right)$ linear absorption coefficients at 388 and $776 \mathrm{~nm}$. Sample 4 is iron doped; the iron concentration $c_{\mathrm{Fe}}$ is about $5.6 \times 10^{19} \mathrm{~cm}^{-3}$ and the linear absorption coefficient $\alpha_{0}$ at $388 \mathrm{~nm}$ is about $15 \mathrm{~cm}^{-1}$. For all the samples and wavelengths used, the linear absorption is negligibly smalli.e., $\alpha_{0} d \ll 1$.

\section{PUMP-PULSE PROPAGATION UNDER STRONG TWO-PHOTON ABSORPTION}

Figure 2 shows the pump transmission coefficient $T_{p}$-i.e., the fraction of the fs pulse that is transmitted. In this experiment just single pulses of $388 \mathrm{~nm}$ wavelength are employed. The $T_{p}$ values are plotted versus the peak pump pulse

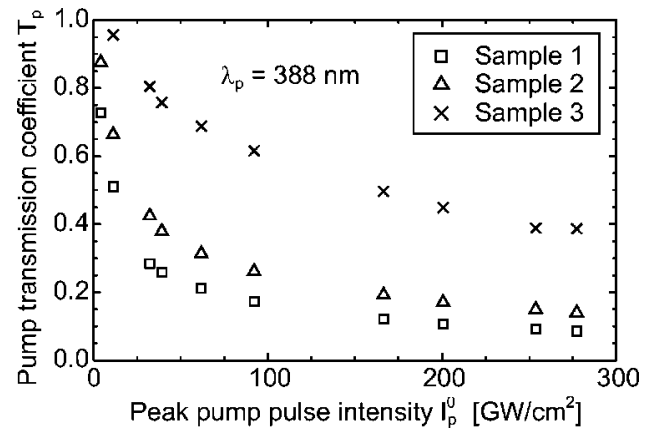

FIG. 2. Transmission coefficient $T_{p}$ versus peak pump pulse intensity $I_{p}^{0}$. The squares, triangles, and crosses correspond to samples 1,2 , and 3 , respectively.

intensity $I_{p}^{0}$. It is obvious that the pulses are strongly absorbed for high incident intensities. In the following we will see whether these data can be explained assuming that twophoton absorption is the dominating process.

The relevant light absorption coefficient is $\alpha_{p}=\beta_{p} I_{p}$, where $I_{p}$ is the pump intensity and $\beta_{p}$ is the quadratic absorption coefficient at the pump wavelength $\lambda_{p}$; linear absorption is supposed to be negligible.

In the actual case (see Fig. 1), the pump intensity obeys the one-dimensional nonlinear wave equation

$$
\left(\frac{\partial}{\partial x}+\frac{1}{v_{p}} \frac{\partial}{\partial t}\right) I_{p}=-\beta_{p} I_{p}^{2},
$$

where $x$ is the propagation coordinate and $v_{p}=c / n_{p}$ is the pulse velocity with $n_{p}$ being the relevant refractive index. This equation follows from Maxwell's equations under the assumptions of negligible diffraction divergence and frequency dispersion, which are well justified for our experimental conditions. It has to be supplemented by a boundary condition at the input face $x=0$. Here we use a Gaussian input profile

$$
I_{p}(x=0)=I_{p}^{0} \exp \left[-\left(t / t_{p}\right)^{2}-\left(r / r_{p}\right)^{2}\right]
$$

where $I_{p}^{0}$ is the input peak intensity and $r=\left(y^{2}+z^{2}\right)^{1 / 2}$ is the radial coordinate while $r_{p}$ and $t_{p}$ characterize the pump beam radius and the temporal half-width.

One can check straightforwardly that the solution of Eq. (1) meeting the boundary condition (2) is given by

$$
\frac{I_{p}^{0}}{I_{p}}=\beta_{p} I_{p}^{0} x+\exp \left[\frac{\left(t-x v_{p}^{-1}\right)^{2}}{t_{p}^{2}}+\frac{r^{2}}{r_{p}^{2}}\right] .
$$

This relation describes the propagation of the pump pulse with the velocity $v_{p}$, the decrease of the peak intensity, and the change of the spatiotemporal profile. The combination $x-v_{p} t$ that enters this relation can be considered as the spatial variable in the coordinate frame moving with the pulse velocity $v_{p}$. By setting $r=0$ and $v_{p} t=x$ one finds that the peak intensity decreases as $\left(1+\beta_{p} I_{p}^{0} x\right)^{-1}$ with increasing propagation distance $x$. By setting $v_{p} t=x$ we find out how the transverse beam profile is changing with increasing $\beta_{p} I_{p}^{0} x$ product (see Fig. 3). 


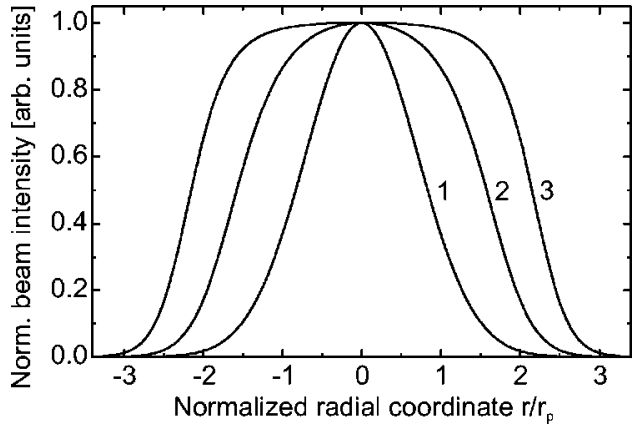

FIG. 3. Transverse beam profile (normalized intensities) for different values of the product $\beta_{p} I_{p}^{0} x$. Lines 1,2 , and 3 are plotted for $\beta_{p} I_{p}^{0} x=0$ (the input shape), 10 , and 100 .

A strong flattening of the top of the pulse is evident; it is caused by the preferential light absorption at the beam center. The same flattening takes place for the temporal shape of the pulse at $r=0$.

Next we calculate the main measurable characteristic of the pump transmission-the normalized transmission coefficient $T_{p}$. It is defined as the ratio of the output pulse energy, measured by the photodetector (see Fig. 1), to the input pulse energy (reflections from the input and output faces can, when necessary, be taken into account). By integrating the output intensity $I_{p}(d, r, t)$ over the time and the transverse coordinates $y, z$ and dividing the quantity calculated by its value at $d=0$, we obtain

$$
T_{p}=\frac{2}{q_{p} \sqrt{\pi}} \int_{0}^{\infty} \ln \left(1+q_{p} e^{-s^{2}}\right) d s
$$

The transmission coefficient depends only on a single nonlinear absorption parameter $q_{p}=\beta_{p} I_{p}^{0} d$; for $q_{p}=0$ and $\infty$, we have $T_{p}=1$ and 0 , respectively. The solid line in Fig. 4 shows the calculated dependence $T_{p}\left(q_{p}\right)$. After a rapid initial fall, the further decrease of $T_{p}$ occurs very slowly; this is indeed due to the relatively weak energy absorption at the pulse wings. Even for an extremely high nonlinear absorption pa-

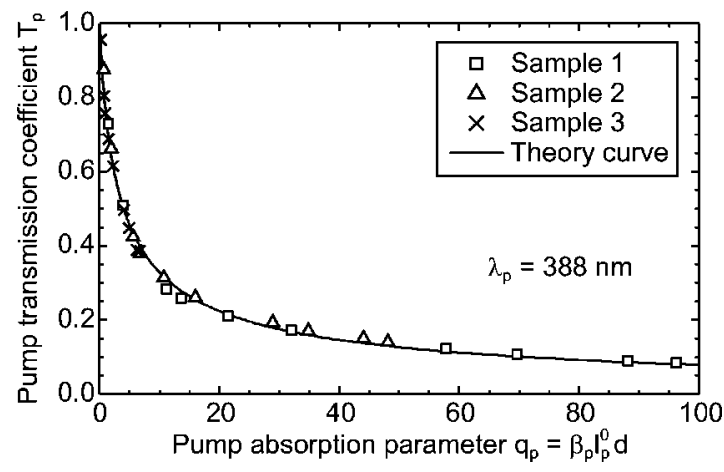

FIG. 4. Dependence of the transmission coefficient $T_{p}$ on $\beta_{p} I_{p}^{0} d$. The solid line (characteristic curve) is calculated from Eq. (4). The squares, triangles, and crosses represent the experimental data on $T_{p}\left(I_{p}^{0} d\right)$, scaled by a factor of $\beta_{p}=3.5 \mathrm{~cm} / \mathrm{GW}$, for samples 1,2 , and 3 , respectively. rameter $q_{p}=100$, about $8 \%$ of the incident energy is passing through.

Now we turn back to the experimental results shown in Fig. 2. In Fig. 4 they are shown again, but now plotted versus the dimensionless pump absorption parameter $q_{p}=\beta_{p} I_{p}^{0} d$. The only variable parameter to fit the characteristic theoretical curve to all experimental data is the two-photon absorption coefficient $\beta_{p}$. An excellent agreement between theory and experiment for all three samples within the large intensity range is evident. This proves unambiguously that two-photon absorption is the reason for the pump attenuation.

The value of the two-photon absorption coefficient at $388 \mathrm{~nm}$ deduced from our experiment is $\beta_{p} \simeq 3.5 \mathrm{~cm} / \mathrm{GW}$. The maximum value of the product $\beta_{p} I_{p}^{0} d$ is about 96, 48, and 7 for samples 1,2, and 3, respectively; the pump pulse is subjected to a strong nonlinear absorption even in the relatively thin samples.

In the general case, the nonlinear absorption coefficient $\beta_{p}$ is expected to be different for the $Z$ - and $Y$-polarized pump beam. Our experiments have shown, however, that the corresponding difference is fairly small, less than $15 \%$.

By varying the pump wavelength, it is possible to measure the dependence $\beta_{p}\left(\lambda_{p}\right)$. The corresponding data for the range $(388-776 \mathrm{~nm})$ are presented elsewhere [21]. As might be expected, the absorption coefficient $\beta_{p}$ is strongly decreasing with increasing pump wavelength; it tends to zero when approaching the upper boundary of the above spectral interval.

Having understood the nonlinear pump propagation, we can now turn to studies of the influence of the pump on the probe pulse propagation.

\section{PROBE TRANSMISSION VERSUS PUMP-PROBE DELAY TIME}

\section{A. Experimental data}

Figure 5 shows the dependence of the probe transmission coefficient $T_{r}$ at $\lambda_{r}=776 \mathrm{~nm}$ on the time delay $\Delta t$ between readout (probe) and pump pulses on the picosecond time scale for sample $4(d=0.07 \mathrm{~mm})$ and four different values of the pump intensity; both pulses are $Y$ polarized (ordinary waves). In all experimental curves $\Delta t$ is defined to be zero when $T_{r}$ reaches the smallest value.

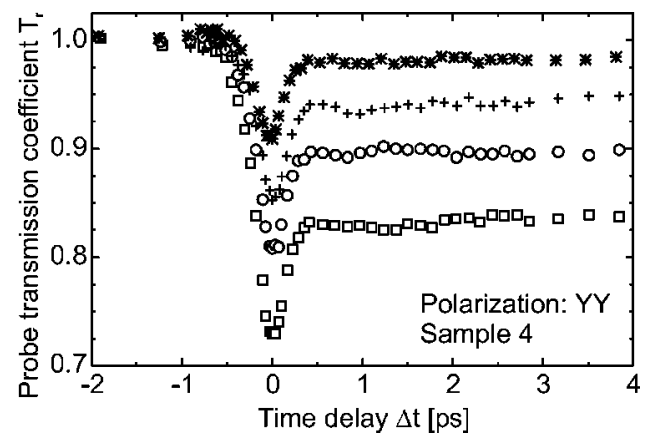

FIG. 5. Normalized transmission coefficient $T_{r}$ for the probe pulse versus the time delay $\Delta t$ for sample 4 and four different values of the pump intensity. The stars, crosses, circles, and squares correspond to $I_{p}^{0} \simeq 43,68,93$, and $139 \mathrm{GW} / \mathrm{cm}^{2}$, respectively. 


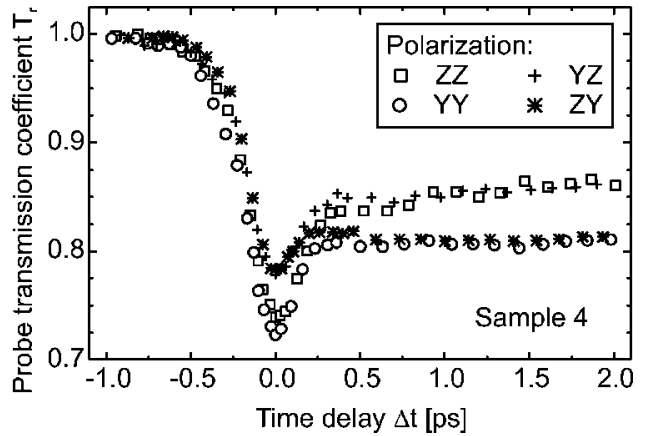

FIG. 6. Transmission coefficient $T_{r}$ versus $\Delta t$ for different pump and probe polarizations; the first and second characters of each pair in the inset (e.g., $Y Z$ ) specify the orientation of the polarization vector of the pump and probe pulses, respectively.

This dependence is highly remarkable. It is characterized by a narrow dip (its temporal width is comparable with that of the pulses) followed by a quasipermanent section (plateau). Both the minimum and plateau values of $T_{r}(\Delta t)$ decrease nonlinearly with increasing pump intensity. One can see also that the decrease of the plateau $T_{r}^{\text {plat }}$ with increasing $I_{p}^{0}$ occurs faster than that of the minimum value $T_{r}^{\min }$.

The influence of the pump and probe polarizations on the probe transmission is illustrated by Fig. 6. It shows the dependence $T_{r}(\Delta t)$ for sample $4, I_{p}^{0} \simeq 105 \mathrm{GW} / \mathrm{cm}^{2}$, and four different combinations of beam polarizations, including the cases of orthogonal polarizations.

The dip is more pronounced for parallel polarization of the pump and probe pulses ( $Z Z$ and $Y Y$ ); the influence of the orientation of the polarization plane is fairly weak. The plateau value of $T_{r}(\Delta t)$ is determined by the probe polarization; it is slightly larger for the $Z$-polarized probe beam.

In relatively thick samples the dependence $T_{r}(\Delta t)$ experiences distortions (see Fig. 7). The dip is noticeably asymmetric in this case and its temporal width is larger than that in Fig. 5. Despite the fact that the maximum value of $q_{p}$ is about 60 in Fig. 7, the value $T_{r}^{\text {min }} \simeq 0.66$ is not much smaller than that in the case of $q_{p} \simeq 4\left(T_{r}^{\min } \simeq 0.74\right)$ (see the data of Fig. 6).

Last, we have measured the dependences of the dip and plateau amplitudes on the pump absorption parameter $\beta_{p} I_{p}^{0} d$.

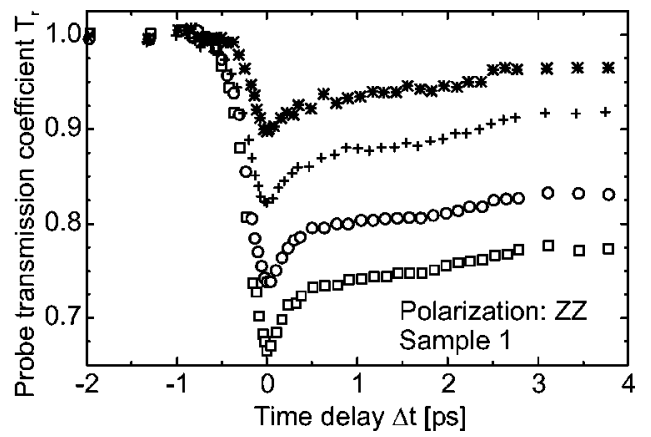

FIG. 7. Normalized transmission coefficient $T_{r}$ for the probe pulse versus the time delay $\Delta t$ for sample $1(d=1 \mathrm{~mm})$ and four different values of the pump intensity. The stars, crosses, circles, and squares correspond to $I_{p}^{0} \simeq 24,49,101$, and $147 \mathrm{GW} / \mathrm{cm}^{2}$, respectively.

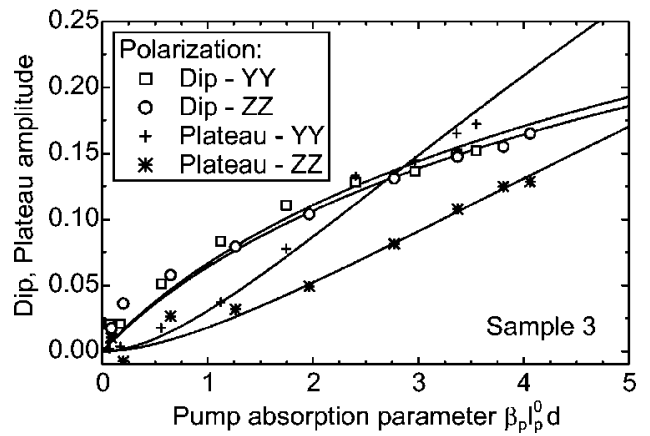

FIG. 8. Dependence of the dip and plateau amplitudes on the product $\beta_{p} I_{p}^{0} d$ for sample 3 and two different pump-probe polarization states. The points are experimental data and the solid lines are theoretical fits.

It was done for thin $(70 \mu \mathrm{m})$ samples where the dip shape is symmetric. The dip and plateau amplitudes are determined as $1-T_{r}^{\min }-\frac{1}{2}\left[1-T_{r}(\Delta t=2 \mathrm{ps})\right]$ and $1-T_{r}(\Delta t=2 \mathrm{ps})$, respectively.

The points in Fig. 8 show the corresponding experimental data for two different polarization cases $Y Y$ and $Z Z$. One sees that the dip amplitude tends to saturate with increasing $\beta_{p} I_{p}^{0} d$ while the plateau amplitude shows no saturation. The solid lines in Fig. 8 show a theoretical fit (see the next two subsections for details).

\section{B. Modeling of probe transmission}

The plateau section in the dependence $T_{r}(\Delta t)$ can be attributed to the absorption of the probe pulse by charge carriers (electrons and/or holes) that are excited via the pumpinduced two-photon process. Recombination of the photoexcited carriers is not expected to occur on the picosecond time scale $[1,22]$. Some polarization dependence of the plateau value is explained by the crystal anisotropy.

The dip in the $T_{r}(\Delta t)$ curve represents a new effect. It contrasts with the effect of increasing probe intensity owing to coherent (parametric) pump-probe interactions which is known for a number of semiconductors [23-25]. The decrease of the probe pulse intensity in the presence of the pump pulse is obviously of a different origin. We attribute it to direct band-band transitions involving simultaneously pump and probe photons; their total energy $(\simeq 4.8 \mathrm{eV})$ exceeds the width of the forbidden gap of $\mathrm{LiNbO}_{3}(\simeq 3.9 \mathrm{eV})$. It is not surprising that identical polarizations of the pump and probe facilitate this two-photon process.

Since the dip and plateau are of different physical origins, we model them separately.

\section{Shape of the dip}

The instantaneous contribution to the pump-induced absorption at the probe wavelength $\lambda_{r}$ can be characterized by the absorption coefficient $\alpha_{r}=\beta_{r} I_{p}$, where $\beta_{r}$ is a new twophoton coefficient. The relevant linear evolution equation for the probe intensity $I_{r}$ has the form [compare to Eq. (1)] 


$$
\left(\frac{\partial}{\partial x}+\frac{1}{v_{r}} \frac{\partial}{\partial t}\right) I_{r}=-\beta_{r} I_{r} I_{p}
$$

where $v_{r}=c / n_{r}$ is the probe pulse velocity and $n_{r}$ is the refractive index at $\lambda_{r}$. Since $I_{r} \ll I_{p}$, the probe cannot influence the pump intensity distribution $I_{p}(x, r, t)$; the latter is given by Eq. (3).

To solve Eq. (5), it is useful to transfer from $x$ and $t$ to the independent variables $x^{\prime}=x$ and $t^{\prime}=t-x v_{r}^{-1}$; this means the transition into a coordinate frame moving with the probe pulse velocity. In the new variables Eq. (5) simplifies to

$$
\partial I_{r} / \partial x^{\prime}=-\beta_{r} I_{r} I_{p}
$$

After simple calculations we obtain from here the following explicit relation for the output intensity of the probe pulse $I_{r}^{d}=I_{r}(d, t, r)$ (in the $x, t$ terms):

$$
I_{r}^{d}=I_{r}\left(0, t-t_{0}, r\right) \exp \left[-\beta_{r} \int_{0}^{d} I_{p}\left(x, t-t_{0}, r\right) d x\right],
$$

where $t_{0}=d / v_{r}$ is the probe pulse traveling time and $I_{r}(0, t, r)$ is the input intensity for the probe.

Let the input profile of the probe beam be given by

$$
I_{r}(0, t, r)=I_{r}^{0} \exp \left[-\frac{(t-\Delta t)^{2}}{t_{p}^{2}}-\frac{r^{2}}{r_{p}^{2}}\right],
$$

where $\Delta t$ is the delay time between probe and pump pulses. It is assumed, in accordance with the experimental conditions, that the probe pulse duration and transverse width at the input are the same as those of the pump, compare with Eq. (2).

Using Eq. (3) and integrating $I_{r}(d, t, r)$ over the time $t$ and the transverse coordinates $y, z$, one can calculate the normalized transmission coefficient of the probe pulse $T_{r}$. The shape of the function $T_{r}(\Delta t)$ is controlled by three dimensionless parameters: the known pump absorption parameter $q_{p}$ $=\beta_{p} I_{p}^{0} d$, the ratio $\beta_{r} / \beta_{p}$, and $\delta_{0}=\left(v_{p}^{-1}-v_{r}^{-1}\right) d / t_{p}$ $=\left(n_{p}-n_{r}\right) d / c t_{p}$. The last parameter is responsible for the broadening and asymmetric distortions of the dip. In $\mathrm{LiNbO}_{3}$, the difference $n_{p}-n_{r}$ is $\simeq 0.2$ for the ordinary waves (the $Y Y$ case) and $\simeq 0.17$ for the extraordinary waves (the $Z Z$ case) [26]. In both polarization cases, the parameter $\delta_{0}$ can be estimated as $1 / 3$ for our thin $(0.07 \mathrm{~mm})$ samples and 5 for our thick $(1 \mathrm{~mm})$ sample.

We consider first the dip shape for the thin samples where the effects of broadening and distortion can be neglected in the leading approximation. By setting $v_{p}=v_{r}$, one can obtain from Eq. (7), after intermediate integrations,

$$
T_{r}=\frac{e^{-\left(\Delta t / t_{p}\right)^{2}}}{\sqrt{\pi} a q_{p}} \int_{-\infty}^{\infty} e^{2 s \Delta t / t_{p}}\left[\left(1+q_{p} e^{-s^{2}}\right)^{a}-1\right] d s,
$$

where $a=\left(\beta_{p}-\beta_{r}\right) / \beta_{p}$. As follows from here, $T_{r}$ is an even function of $\Delta t$; i.e., the dip shape is symmetric.

In the limit of weak pump absorption, $q_{p} \ll 1$, Eq. (9) transforms into the following simple relation:

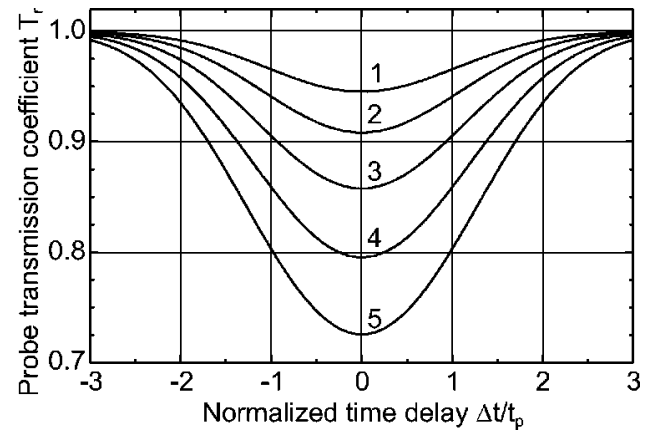

FIG. 9. The normalized transmission coefficient $T_{r}$ versus $\Delta t / t_{p}$ for $\beta_{r} / \beta_{p}=0.2$ and $q_{p}=1,2,4,8$, and 16 (curves $1,2,3,4$, and 5, respectively).

$$
T_{r} \simeq 1-\frac{\beta_{r} I_{p}^{0} d}{2 \sqrt{2}} \exp \left[-\frac{(\Delta t)^{2}}{2 t_{p}^{2}}\right]
$$

Thus we have a Gaussian-shaped dip for $T_{r}(\Delta t)$. Its temporal width is larger than that of the input pulses by a factor of $\sqrt{2}$. The amplitude of the $\operatorname{dip}, 1-T_{r}(0)$, grows linearly with increasing pump intensity $I_{p}^{0}$ and probe absorption coefficient $\beta_{r}$.

Unfortunately, the field of applicability of the approximation of weak pump absorption is rather narrow. If $q_{p} \gtrsim 1$, the dip amplitude depends nonlinearly on $I_{p}^{0}$. Figure 9 shows the dependence of $T_{r}$ on the normalized delay time $\Delta t / t_{p}$ plotted on the basis of Eq. (9) for $q_{p}=1,2,4,8$, and 16; the ratio $\beta_{r} / \beta_{p}$ is set to be equal 0.2 . One can see that the width of the dip gradually becomes larger with increasing $\beta_{p} I_{p}^{0} d$ product. The shape of the dip is not Gaussian in the general case.

The dependence of the dip amplitude on the pump absorption parameter $q_{p}$ is shown in Fig. 10 for four representative values of the ratio $\beta_{r} / \beta_{p}$. This dependence is linear only for $q_{p} \lesssim 1$; outside this range the dip amplitude experiences a strong saturation. The amplitude of the dip grows noticeably with increasing $\beta_{r} / \beta_{p}$ ratio. Since the coefficient $\beta_{p}$ is known from the pump absorption experiments, it is possible to estimate the value of $\beta_{r}$ from the pump-probe experiments (see below).

Consider last the effects of broadening and distortion of the dip caused by the difference in the pump and probe velocities; these effects are expected to be significant for our

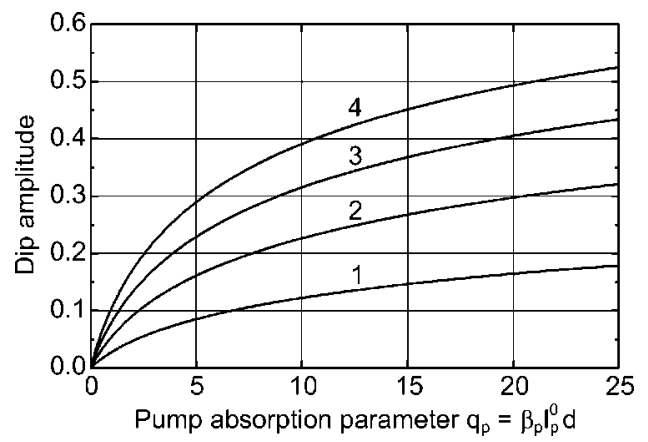

FIG. 10. Dependence of the dip amplitude on $q_{p}$; lines 1, 2, 3, and 4 are plotted for $\beta_{r} / \beta_{p}=0.1,0.2,0.3$, and 0.4 , respectively. 


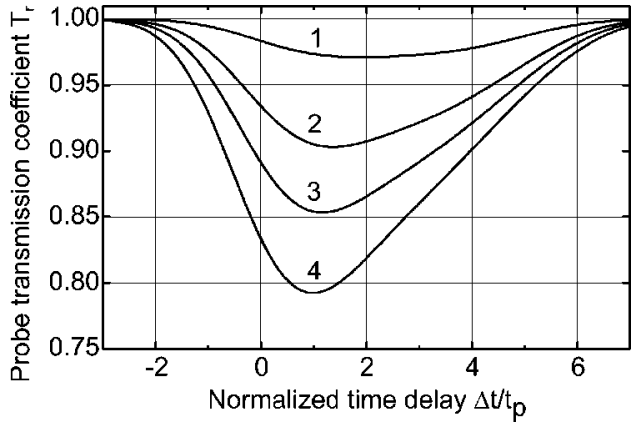

FIG. 11. The normalized transmission coefficient $T_{r}$ versus $\Delta t / t_{p}$ for $d=1 \mathrm{~mm}, \beta_{r} / \beta_{p}=0.2$, and $\delta_{0}=5$. Curves $1,2,3$, and 4 are plotted for $q_{p}=1,5,10$, and 20 , respectively.

thick ( $1 \mathrm{~mm})$ sample. By keeping $v_{p} \neq v_{r}$ in Eq. (7), one can obtain the general relation

$$
\begin{aligned}
T_{r}= & \frac{1}{\sqrt{\pi}} \int_{-\infty}^{\infty} \exp \left[-\left(s-\Delta t / t_{p}\right)^{2}\right] \\
& \times \int_{0}^{1} \exp \left[-\frac{\beta_{r}}{\beta_{p}} \int_{0}^{1} \frac{q_{p} f d u}{q_{p} f u+\exp \left[\left(s-\delta_{0} u\right)^{2}\right]}\right] d f d s .
\end{aligned}
$$

In the limiting case $\delta_{0}=0$ two of three integrations can be performed analytically and we return to Eq. (9).

Figure 11 shows the shape of the dip for $\delta_{0}=5$ and several values of the pump intensity. One sees that the dip experiences a noticeable broadening and becomes asymmetric, its right wing being considerably longer than the left one. Qualitatively, these features are in good agreement with the experimental data for the 1-mm sample presented in Fig. 7. Furthermore, the dip amplitude is noticeably smaller in the thick sample under the condition that the values of $\beta_{p} I_{p}^{0} d$ be the same for the thin and thick crystals. This feature is also in line with our experimental data (compare Figs. 5 and 7). Note that according to Fig. 11 the minimum of the transmission can occur after $\Delta t / t_{p}=0$. This is not in contradiction to our experiment (e.g., Fig. 7), because there $\Delta t / t_{p}$ was set to be zero at the minimum of the transmission.

\section{Plateau amplitude}

Since the pump excites carriers via direct two-photon transitions, it is able to induce an additional absorption for the probe. This pump-induced absorption is expected to remain until recombination of the photoexcited carriers occurs-i.e., at least on the picosecond scale.

We suppose that the two-photon absorption of the pump pulse results in generation of electron-hole pairs. The generation rate is $\alpha_{p} I_{p} / 2 \hbar \omega_{p}=\beta_{p} I_{p}^{2} / 2 \hbar \omega_{p}$, where $\hbar \omega_{p}$ is the energy of a single pump quantum. If $N$ and $P$ are the concentrations of photoexcited electrons and holes, respectively, we have, in terms of $x^{\prime}, t^{\prime}$ (in the moving coordinate frame),

$$
\frac{\partial N}{\partial t^{\prime}}=\frac{\partial P}{\partial t^{\prime}}=\frac{\beta_{p} I_{p}^{2}}{2 \hbar \omega_{p}}=-\frac{1}{2 \hbar \omega_{p}} \frac{\partial I_{p}}{\partial x^{\prime}} .
$$

Recombination processes are neglected in these equations.

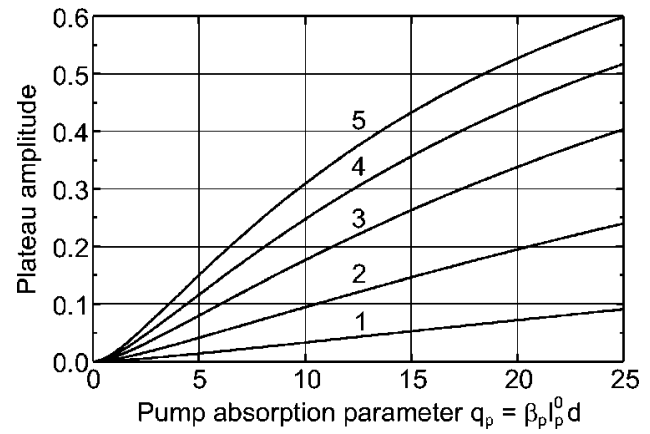

FIG. 12. Dependence of the plateau amplitude on the pump absorption parameter $q_{p}$; lines $1,2,3,4$, and 5 are plotted for $b$ $=0.01,0.03,0.06,0.09$, and 0.12 , respectively.

In accordance with Eq. (12), the free-carrier contribution to the absorption coefficient for the probe $\alpha_{r}^{f}$ can be represented as

$$
\alpha_{r}^{f}\left(x^{\prime}, t^{\prime}\right)=-\frac{\sigma_{r}}{\hbar \omega_{p}} \frac{\partial}{\partial x^{\prime}} \int_{-\infty}^{t^{\prime}} I_{p}\left(x^{\prime}, t^{\prime \prime}\right) d t^{\prime \prime},
$$

where $\sigma_{r}$ is the effective absorption cross section of the photoexcited carriers at the readout wavelength $\lambda_{r}$. Since the function $I_{p}\left(x^{\prime}, t^{\prime}\right)$ is known [see Eq. (3)] it is possible to calculate the above integral.

The probe intensity $I_{r}\left(x^{\prime}, t^{\prime}\right)$ obeys the equation $\partial I_{r} / \partial x^{\prime}$ $=-\left(\beta_{r} I_{p}+\alpha_{r}^{f}\right) I_{r}$ [compare to Eq. (5)]. Its solution has the form

$$
I_{r}=I_{r}\left(0, t^{\prime}\right) \exp \left[-\int_{0}^{x^{\prime}}\left(\beta_{r} I_{p}+\alpha_{r}^{f}\right) d x^{\prime \prime}\right]
$$

The time dependence of $I_{r}$ comes from the dependence of $\alpha_{r}^{f}$, $I_{p}$, and $I_{r}(x=0)$ on $t^{\prime}$.

Consider the case when the pump pulse has passed through; i.e., the instantaneous processes are over. This corresponds to the plateau value of the probe transmission. Mathematically, we have to set $I_{p}=0$ in Eq. (14) and $t^{\prime}=\infty$ in Eq. (13); the value of $\alpha_{r}^{f}$ depends then only on $x^{\prime}$ (and $r$ ) but does not depend on $t^{\prime}$. Using Eq. (8) for $I_{r}(0, t, r)$, it is not difficult now to calculate the plateau value of the transmission coefficient $T_{r}^{\text {plat }}$,

$$
T_{r}^{p l a t}=\int_{0}^{\infty} \exp \left[-f-b q_{p}\left(\frac{\sqrt{\pi}}{2} e^{-f}-\int_{0}^{\infty} \frac{d s}{q_{p}+e^{f+s^{2}}}\right)\right] d f,
$$

where $b=2 \sigma_{r} t_{p} / \hbar \omega_{p} \beta_{p} d$ is a new dimensionless parameter. The value of $T_{r}^{\text {plat }}$ depends on two characteristic parameters $q_{p}$ and $b$; only the first of them depends on the pump intensity $I_{p}^{0}$. Since the decrease of $T_{r}$ is relatively small in the experiments and the parameter $q_{p}$ is definitely larger than unity, it is reasonable to expect that $b \ll 1$.

Lines $1-5$ in Fig. 12 show the dependence of the plateau amplitude, $1-T_{r}^{\text {plat }}$, on the pump absorption parameter $q_{p}$ $=\beta_{p} I_{p}^{0} d$ for five representative values of $b$. This amplitude grows quadratically for $q_{p} \lesssim 1$ and almost linearly for $1 \lesssim q_{p} \lesssim 15$. Its saturation with increasing $q_{p}$ occurs rather 
slowly. The behavior of the dip amplitude within the same interval (see Fig. 10) is different. Since the value of $q_{p}$ is known from pump-absorption experiments, it is possible to estimate $b$ and the cross section $\sigma_{r}$ from the data of pumpprobe experiments.

\section{Comparison with experimental results and determination of probe absorption parameters}

In accordance with the theory, our experiment shows that the dip profile is almost symmetric in the thin $(70 \mu \mathrm{m})$ samples (see Figs. 5 and 9). According to the experiments, the ratio FWHM of the dip / FWHM of pump pulse ranges from $\simeq 1.7$ to $\simeq 2.1$ for the thin samples. The theory predicts the range 1.4-1.6 for this ratio. The difference is not large. It can be explained by an uncertainty of the measurements and some contribution originating from different pump and probe velocities. Considerable broadening of the dip and the noticeable asymmetry of its shape in the 1-mm sample, observed experimentally (see Fig. 7), are in a good qualitative agreement with theory (Fig. 11).

Figure 8 shows experimental data for the dependence of the dip and plateau amplitudes on the pump absorption parameter $q_{p}$, obtained for two different polarization cases, together with the theoretical fit. The fit parameters are the ratio $\beta_{r} / \beta_{p}$ (for the dip amplitude) and $b=2 \sigma_{r} t_{p} / \hbar \omega_{p} \beta_{p} d$ (for the plateau amplitude). The agreement between theory and experiment is pretty good. Whereas the dip amplitude experiences a remarkable saturation with increasing $q_{p}$, the plateau amplitude shows no saturation within the experimental intensity range.

The ratio $\beta_{r} / \beta_{p}$ deduced from the fit procedure is $\simeq 0.245$ for the $Y Y$ case and $\simeq 0.235$ for the $Z Z$ case. Thus, with an accuracy of (10-15)\%, the value of $\beta_{r}$ can be estimated as $\simeq 0.88 \mathrm{~cm} / \mathrm{GW}$. The values of $b$ can be estimated as $\simeq 0.237$ and $\simeq 0.138$ for the $Y Y$ and $Z Z$ cases, respectively. This gives, for the excitation cross section, $\sigma_{y y} \simeq 9.2 \times 10^{-18} \mathrm{~cm}^{2}$ and $\sigma_{z z} \simeq 6.0 \times 10^{-18} \mathrm{~cm}^{2}$. With an accuracy of $\simeq 10 \%$ the above estimates are applicable to sample 3 .

\section{DISCUSSION}

Below we discuss two important issues that are closely related to the results obtained.

General physical picture. According to the data of our collinear pump-probe experiments, the action of strong femtosecond pulses at $388 \mathrm{~nm}$ on $\mathrm{LiNbO}_{3}$ meets the following physical picture.

(i) Initially, an instantaneous generation of free carriers takes place owing to direct two-photon processes. The corresponding nonlinear absorption coefficient is pretty high, $\beta_{p} \simeq 3.5 \mathrm{~cm} / \mathrm{GW}$, so that the pump pulse experiences a strong attenuation even in relatively thin samples.

(ii) The pump-excited carriers do not experience recombination to the levels where they originate from at least on the time scale of tens of picoseconds. These carriers are responsible for the quasipermanent probe absorption (absorption plateau) at $776 \mathrm{~nm}$.

(iii) When a weak probe pulse at $776 \mathrm{~nm}$ is overlapping (in time and space) with a strong pump pulse, an additional instantaneous probe absorption, involving pump and probe photons, takes place. This nonlinear absorption causes a pronounced dip in the dependence of the probe transmission on the delay time between pump and probe pulses. The relevant nonlinear absorption coefficient is also pretty high, $\beta_{r}$ $\simeq 0.88 \mathrm{~cm} / \mathrm{GW}$.

Carrier relaxation. What happens with free carriers excited by pump pulses into the conduction and valence bands and what is the nature of carriers responsible for the plateau in the probe absorption?

The energy of two pump quanta, $2 \hbar \omega_{p} \simeq 6.4 \mathrm{eV}$, exceeds noticeably the energy gap of $\mathrm{LiNbO}_{3}$ crystals $(\simeq 3.9 \mathrm{eV})$. Therefore the band energy of photoexcited hot carriers exceeds considerably the thermal energy, $k_{B} T \simeq 0.026 \mathrm{eV}$. At the first steps of establishment of the thermal equilibrium, energy relaxation and thermalization of hot carriers take place. The characteristic time of this process lies typically in the range $\left(10^{-13}-10^{-12}\right) \mathrm{s}[1,22]$. When the energy relaxation is over, the electrons (holes) occupy energy levels at the bottom (top) of the conduction (valence) band and/or shallow levels in the close vicinity of these bands. The subsequent recombination occurs much slower, typically during the time $\geq 10^{-9} \mathrm{~s}$. Details of the final relaxation stage depend greatly on the concentration and position of the energy levels in the forbidden energy gap. These levels can originate from doping and from partial disorder of the crystal structure.

The data $T_{r}(\Delta t)$ obtained in the thin samples (see Fig. 5) show no indication of an energy relaxation of hot carriers. No new temporal features with a characteristic scale essentially different from the pulse duration can be seen. This means that either the probe absorption cross section does not depend on the carrier energy or the time of energy relaxation is shorter than the pulse duration. The second possibility looks most probable and it does not contradict the above general estimates. If it is the case, shorter pulses are needed to monitor the energy relaxation. The asymmetry of the dip shape in the thick sample (see Fig. 7), which might, by itself, be interpreted as the effect of energy relaxation, is explained by the difference in the pump and probe velocities.

Most probably the pump-excited carriers, which are responsible for the plateau section of the probe transmission, occupy shallow levels near the conduction band. The presence of such levels, attributed usually to the structural $\mathrm{Nb}_{\mathrm{Li}}^{4+}$ defects of $\mathrm{LiNbO}_{3}$ (small polarons), is known from pulse experiments on light-induced infrared absorption [20,27]. Recombination of such excited localized electrons occurs on the time scale $10^{-8}-10^{-5} \mathrm{~s}$ and follows the stretchedexponential law typical for disordered materials.

\section{CONCLUSIONS}

Two-photon absorption processes strongly affect the propagation of high-power femtosecond pump pulses in $\mathrm{LiNbO}_{3}$ at $388 \mathrm{~nm}$. The corresponding nonlinear absorption coefficient, $\beta_{p} \simeq 3.5 \mathrm{~cm} / \mathrm{GW}$, does not show a strong dependence on light polarization and crystal doping.

Strong pump pulses induce instantaneous and quasipermanent contributions to probe absorption at $776 \mathrm{~nm}$. The 
instantaneous contribution is due to two-photon processes with participation of pump and probe photons; the corresponding nonlinear absorption coefficient $\beta_{r} \simeq 0.88 \mathrm{~cm} / \mathrm{GW}$ is not polarization sensitive. The quasipermanent contribution is due to probe absorption by pump-excited carriers; the absorption cross section $\sigma_{r}$ is about $9.2 \times 10^{-18} \mathrm{~cm}^{2}$ and $6.0 \times 10^{-18} \mathrm{~cm}^{2}$ for the light polarization vector perpendicular and parallel to the polar axis, respectively.

The time of hot-carrier relaxation is expected to be shorter than $0.1 \mathrm{ps}$ for the excitation wavelength $388 \mathrm{~nm}$.

Strong nonlinear absorption effects will be important also for femtosecond pulse recording of spatial gratings in $\mathrm{LiNbO}_{3}$.

\section{ACKNOWLEDGMENT}

Financial support from the Deutsche Telekom AG, from the Deutsche Forschungsgemeinschaft (Award No. BU 913/ 13-1), and from the NSF (Engineering Research Centers Program for Neuromorphic Systems Engineering under Award No. EEC-9402726, NSF-Germany Cooperative Research Grant No. INT-0233988) is gratefully acknowledged.
[1] A. Othonos, J. Appl. Phys. 83, 1789 (1998).

[2] A. McClelland, V. Fomenko, and E. Borquet, J. Phys. Chem. B 108, 3789 (2004).

[3] T. Sjodin, H. Petek, and H. L. Dai, Phys. Rev. Lett. 81, 5664 (1998).

[4] R. DeSalvo, A. A. Said, D. J. Hagan, E. W. Van Strylnd, and M. Sheik-Bahae, IEEE J. Quantum Electron. QE-32, 1324 (1996).

[5] A. Dragonmir, J. G. Mclnerney, and D. N. Nikogosyan, Appl. Opt. 41, 4365 (2002).

[6] D. von der Linde, A. M. Glass, and K. F. Rodgers, Appl. Phys. Lett. 25, 155 (1974).

[7] C.-T. Chen, D. M. Kim, and D. von der Linde, Appl. Phys. Lett. 34, 321 (1979).

[8] A. L. Smirl, K. Bohnert, G. C. Valley, R. A. Mullen, and T. F. Boggess, J. Opt. Soc. Am. B 6, 606 (1989).

[9] M. Ewart, I. Biaggio, M. Zgonik, and P. Günter, Phys. Rev. B 49, 5263 (1994).

[10] L. Solymar, D. J. Webb, and A. Grunnet-Jepsen, The Physics and Applications of Photorefractive Materials (Clarendon Press, Oxford, 1996).

[11] K. Buse, Appl. Phys. B: Lasers Opt. 64, 391 (1997).

[12] Holographic Data Storage, edited by H. J. Coufal, D. Psaltis, and G. T. Sincerbox (Springer, Berlin, 2000).

[13] Infrared Holography for Optical Communications, edited by P. Boffi, D. Piccinin, and M. C. Ubaldi (Springer, Berlin, 2003).

[14] M. Centini, C. Sibilia, M. Scalora, G. D’Aguanno, M. Berto- lotti, M. J. Bloemer, C. M. Bowden, and I. Nefedov, Phys. Rev. E 60, 4891 (1999).

[15] V. Leyva, G. A. Rakuljic, and B. O'Conner, Appl. Phys. Lett. 65, 1079 (1994).

[16] M. M. Fejer, G. A. Magel, D. H. Jundt and R. L. Byer, IEEE J. Quantum Electron. 28, 2631 (1992).

[17] R. Müller, M. T. Santos, L. Arizmendi, and J. M. Cabrera, J. Phys. D 27, 241 (1994).

[18] S. Breer and K. Buse, Appl. Phys. B: Lasers Opt. 66, 339 (1998).

[19] D. N. Nikogosyan, Properties of Optical and Laser-Related Materials (Wiley, New York, 1997).

[20] F. Jermann and J. Otten, J. Opt. Soc. Am. B 10, 2085 (1993).

[21] O. Beyer, D. Maxein, K. Buse, B. Sturman, H. T. Hsieh, and D. Psaltis, Opt. Lett. 30, 1366 (2005).

[22] K. Seeger, Semiconductor Physics (Springer, New York, 1973).

[23] H. J. Eichler, P. Günter, and D. W. Pohl, Laser-Induced Dynamic Gratings (Springer-Verlag, Berlin, 1986).

[24] C. J. Kennedy, J. C. Matter, A. L. Smirl, H. Weiche, F. A. Hopf, S. V. Pappu, and M. O. Scully, Phys. Rev. Lett. 32, 419 (1974).

[25] C. V. Shank and D. H. Auston, Phys. Rev. Lett. 34, 479 (1975).

[26] U. Schlarb and K. Betzler, J. Appl. Phys. 73, 3472 (1993).

[27] D. Berben, K. Buse, S. Wevering, P. Herth, M. Imlau, and Th. Woike, J. Appl. Phys. 87, 1034 (2000). 